Article

\title{
Redundant Symmetry Influences Perceptual Grouping (as Measured by Rotational Linkage)
}

\author{
Barbara Gillam \\ School of Psychology, The University of New South Wales, Sydney 2052, Australia; b.gillam@unsw.edu.au; \\ Tel.: +61-2-9385-3522
}

Academic Editor: Marco Bertamini

Received: 8 February 2017; Accepted: 2 May 2017; Published: 9 May 2017

\begin{abstract}
Symmetry detection has long been a major focus of perception research. However, although symmetry is often cited as a "grouping principle", the effect of symmetry on grouping, an important form of perceptual organization, has been little measured. In past research, we found little spatio-temporal grouping for oblique lines symmetric around a horizontal axis during ambiguous rotary motion in depth. Grouping was measured by the degree to which the ambiguous motion direction was resolved for two elements in common (rotational linkage). We hypothesized that symmetry-based grouping would be stronger if symmetry was redundant i.e., carried by elements of greater complexity. Using the rotational linkage measure, we compared grouping for horizontally symmetric simple oblique lines and for lines composed of multiple conjoined orientations and found greater grouping for the more complex symmetric lines. A control experiment ruled out possible confounding factors and also showed a grouping effect of vertically aligned endpoints. We attribute the stronger grouping effect of redundant symmetry to the fact that it has a lower probability than does simple symmetry of arising from an accidental environmental arrangement.
\end{abstract}

Keywords: symmetry; grouping; redundancy; complexity; rotary motion in depth

\section{Introduction}

Symmetry is one of the defining characteristics of Gestalt "good figure" [1]. This implies that symmetric organizations will be preferred to asymmetric ones and that isolated elements will cohere into groups more readily if those elements are symmetric. More recently, Treder and Van der Helm [2] conclude that symmetry is a cue for the presence of one object, which also implies grouping. However, overwhelmingly, symmetry research explores its detection and saliency rather than its influence on perceptual organization, e.g., Barlow and Reeves [3]; Royer [4]; Wenderoth [5]; Tyler [6]; Wagemans [7]; Gurnsey, Herbert and Kenemy [8]. Although Locher and Wagemans [9] investigated the effect of grouping patterns and Bertamini, Friedenberg and Kubovy [10] the effect of contour organization on symmetry detection; the reverse influence has been little explored. Pomerantz and Kubovy [11] argued that it does not follow that because a property such as symmetry has salience, it plays a significant role in the process of organization. However, it has been known since the early work of Bahnsen [12], a student of Rubin [13], that a symmetric shape is more likely to be seen as a figure than an asymmetric one in a figure-ground task. This has been confirmed more recently by Peterson and Gibson [14] and Mojika and Peterson [15], who also showed that the effect of symmetry on figure-ground resolution was enhanced when several alternating symmetric regions were added to the display. Kanizsa and Gerbino [16] reported that convexity overrides symmetry in eliciting perception of figure, leading Pomerantz and Kubovy [11] to argue that since it is so easily overridden, symmetry probably plays little role in organization and only becomes available after elements are organized into units on other grounds. The Mojika and Peterson's [15] study showed, however, that with appropriate controls, symmetry is as effective as convexity. 
Machilsen, Pauwels and Wagemans [17], stated that generally there has been "no certain benefit of symmetry on perceptual grouping" (p. 3), but they did show a small influence of vertical symmetry on the segregation of a pattern from a noisy background. It is well established that vertical symmetry is more salient than horizontal symmetry, a fact that is usually attributed to the ubiquitous vertical symmetry of biological structures. In a rare study extending these salience findings to perceptual organization, Gillam and McGrath [18] showed that two oblique lines arranged with vertical symmetry are grouped more than when arranged with horizontal symmetry and that the operative symmetry axis was retinal not environmental. The grouping criterion that these authors used was "rotational linkage" also used in the present experiments and described below.

The only other research we are aware of which measured the effect of symmetry on perceptual grouping is by Feldman [19] who measured the degree of grouping of two separate straight lines in various arrangements (See Figure 1) among which one pair (Figure 1E) had horizontal symmetry. His grouping criterion was the degree to which a given line pair showed an "object benefit". This is said to be present when comparisons of visual features are faster within a single object than between objects. Feldman found greatest object benefit for the symmetric figure (Figure 1E). However, Gillam [20], who studied the grouping of pairs of lines using the "rotational linkage" criterion, found that an almost identical figure to Feldman's Figure 1E (see Figure 2A) produced little grouping despite its symmetry. In accounting for the difference between the Feldman [19] and Gillam [20] results, it could be argued that the rotational linkage criterion is a more direct measure of grouping than object benefit, which measures a consequence of object formation rather than its determinants. The essence of rotational linkage (a term coined by Eby, Loomis and Solomon, [21]) is to present two or more lines (or objects) rotating in depth in parallel projection, which renders direction of motion (clockwise or counterclockwise) ambiguous (Wallach and $\mathrm{O}^{\prime}$ Connell, [22]). The proportion of total exposure time during which the ambiguous rotation directions are resolved in common for several lines or objects is measured. Observers are asked to hold down a switch whenever individual elements appear to be rotating in opposite directions. Gillam [20] calls this "fragmentation time". Rotational linkage is measured as the total time minus the fragmentation time for a given number of rotations and provides a quantitative measure of grouping.

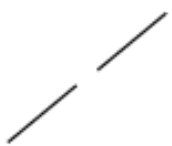

A collinear

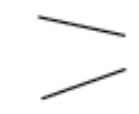

E symmetric

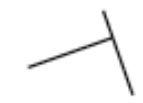

I right angle

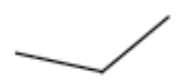

B
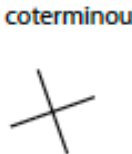

F 2-symmetric

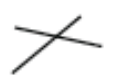

J contact
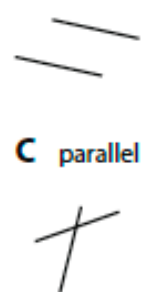

G skewsymmetric

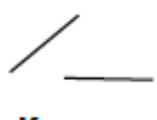

K generic
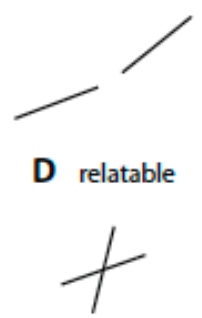

H 2-skewsymmetric

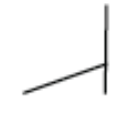

L T-junction

Figure 1. The stimulus figures used by Feldman [19]. See text.

Using the rotational linkage criterion, Gillam and colleagues have shown that the grouping of two lines is influenced by a number of stimulus factors including: (a) relative line orientation (Gillam, [20]); (b) line separation relative to length (Gillam, [23]; Gillam and Grant Jr., [24]); (c) axis of rotation (Gillam and McGrath, [18]); and (d) common vanishing point with a surrounding frame (Gillam and Broughton, [25]). A general discussion of this approach to grouping can be found in Gillam [26]. 
The goal of the present research was to use the rotational linkage criterion to see whether the poor grouping (high rate of fragmentation) that we have generally found for the horizontally symmetric figure (Figure 2A) could be improved by increasing symmetry redundancy, which can be achieved by making the reflected lines more complex. Figure 2A has only two symmetrically arranged oblique straight lines. We compared fragmentation for this figure and for figures in which the horizontally symmetric straight oblique lines are replaced by similarly reflected lines made up of adjoined multiple orientations but with the same average orientation and separation as the lines of Figure 2A. These figures are shown in Figure 2B,C.

Our reason for using horizontal symmetry to investigate the effects of redundancy was that an earlier study (Gillam and McGrath, [18]) showed that Figure 2A turned 90 degrees to create vertical symmetry demonstrated strong rotational linkage leaving little scope for any additional effects of redundancy.

Greater grouping with more redundant symmetry is predictable on the grounds that symmetry in more complex figures is less likely than in simple figures to result from an accidental arrangement of two unrelated elements and is therefore more likely to be seen as a structural property. This outcome would be predicted by Rock's [27] view that perceptual resolutions avoid "coincidence". A similar outcome would follow from a Bayesian analysis (Barlow, [28]) in that more redundantly symmetric figures would have a higher probability of resulting from a distally symmetric arrangement. Symmetry detection on the other hand has been considered by van der Helm [2] to have a holographic structure in which the number of elements is irrelevant. Supporting evidence for this proposition, using blob stimuli, was reported by Csatho, van der Vloed and van der Helm [29]. Additionally, Baylis and Driver [30], using line stimuli more like those in the present study, found that the speed of symmetry detection for the two sides of a single object is not influenced by the complexity of the reflected contour. Finally, in a comprehensive visual evoked response study, Makin et al. [31] confirmed many aspects of the holographic model, including the fact that complexity has little effect on symmetry detection. Our hypothesis that redundant symmetry will facilitate grouping thus seems to be somewhat at odds with the general finding for symmetry detection. However, grouping based on symmetry will not necessarily be determined by the same factors as symmetry detection, which must occur prior to grouping.

\section{Experiment 1}

\subsection{Method}

The three figures used are shown drawn to scale at a quarter actual size in Figure 2. The axis of rotation is indicated by a dotted line. Observers sat at a distance of $140 \mathrm{~cm}$ from the screen with their eyes at the level of the midpoint of the object on the screen. The separation of the lines of Figure $2 \mathrm{~A}$ along the axis of rotation was $2.5 \mathrm{~cm}$ (one degree). The average line orientation (15 degrees from the horizontal) and average separation were the same in all three figures. To achieve smooth motion, figures were presented on a computer-controlled Hewlett-Packard oscilloscope with a fast phosphor. A red filter placed over the screen eliminated faint ghosts caused by persistence. Each figure was presented for 10 rotations at a speed of $12 \mathrm{~s}$ per rotation. This slow smooth rotation has been found in the past to elicit ample opportunities for apparent change from fragmentation (opposite rotation) to rotational linkage. Although strict fixation was not required, a dot to guide location of looking was placed midway between the upper and lower sets of lines on the axis of rotation (which was not shown). 
A

B

C

\section{Mean}

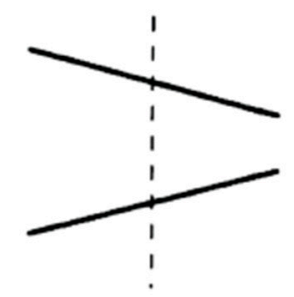

$36.9 \mathrm{sec}$.

$23.9 \mathrm{sec}$.
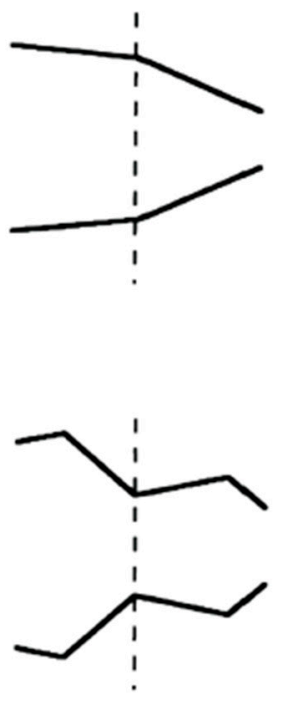

\section{$18.2 \mathrm{sec}$.}

Figure 2. The figures used in Experiment 1 (drawn to one-quarter scale). The mean fragmentation time in seconds is shown next to each figure.

\subsection{Observers}

Participants in both experiments were volunteers from the State University of New York, State College of Optometry. For experiment 1, twelve students, ignorant of the rationale of the experiment, were used. Each observer was shown Figure 2A (known to readily fragment) and asked what he/she saw. As in all our grouping experiments, only those observers who spontaneously reported seeing fragmentation for Figure $2 \mathrm{~A}$ in a pretest were used in the main Experiment. This ensured that they were clear about what they were supposed to respond to. Two of the twelve observers only reported fragmentation after it was suggested to them and were not used in the main experiment. The figures were presented for each observer in a different random order.

\subsection{Results}

The mean fragmentation time is shown next to each figure in Figure 2. An analysis of variance (ANOVA) showed a significant effect of figure ( $F=7.29$, $\mathrm{df} 2,18 p<0.005)$. Post-hoc Newman-Keuls tests showed that the mean for Figure 2A was significantly higher than the means for Figure 2B,C, $(5.3 p<0.01 ; 3.7 p<0.05$ respectively). Although Figure $2 \mathrm{C}$ had a lower mean fragmentation time (more grouping) than Figure 2B as predicted, these two means did not differ significantly from each other on a Newman-Keuls test.

The results support the hypothesis that symmetry carried by more complex elements (made up of multiple line orientations) results in greater grouping than symmetry carried by simple single line elements. It is possible that another difference between the simple and more complex stimuli is responsible rather than symmetry redundancy per se. It is unlikely however that the effect of complex symmetry arose from a fortuitous effect of the particular figures used, since Figure $2 \mathrm{~B}, \mathrm{C}$ differ 
considerably from each other. For example, Figure $2 \mathrm{~B}$ is convex whereas Figure $2 \mathrm{C}$ is both convex and concave yet both enhanced grouping relative to Figure 2A. Experiment 2 explores these issues further.

\section{Experiment 2}

In this experiment, we attempted to replicate the basic finding of Experiment 1 with respect to complexity and to compare the effects of concave and convex symmetry. We also compared fragmentation time for symmetric figures and for similarly complex elements (lines with a mid-line orientation change) but not arranged symmetrically. Finally, in Experiment 2, we investigated the effect on grouping of offsetting the upper element horizontally from the lower element. This destroyed any reflective symmetry present while maintaining the same upper and lower elements as in the symmetric figures. This manipulation also explored vertical alignment of contour end points as a grouping factor.

\subsection{Method}

The figures used in Experiment 2 are shown drawn in Figure 3 at one-third actual size. There were four basic figures, each composed of an upper and lower element with endpoints vertically aligned and the same four figures with the upper and lower elements offset. The four basic figures were Figure $3 \mathrm{~A}$ (Figure 2A from Experiment 1), Figure 3B (Figure 2B from Experiment 1, which was symmetric about a central horizontal axis and convex), Figure $3 \mathrm{C}$ (A concave figure symmetric about a central horizontal axis, which consisted of two lines per element that had the same average orientation and spacing as Figure 3A,B) and Figure 3D (A composite figure consisting of one line from Figure 3B and one from Figure $3 \mathrm{C}$ ). Figure 3D provided a baseline non-symmetric figure against which to compare the symmetric Figure 3B,C from which its components were drawn. Finally, Figure 3E-H were the offset versions of the four basic Figure 3A-D respectively. These have the same components as the equivalent non-offset figures but lack symmetry.

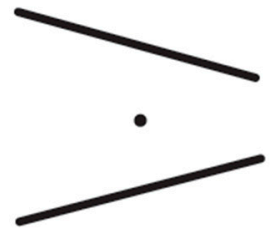

A

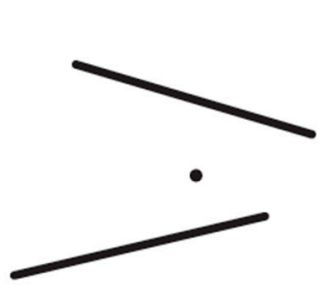

E

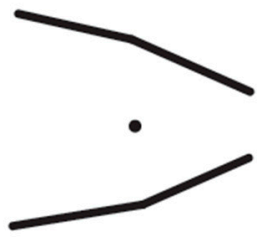

B

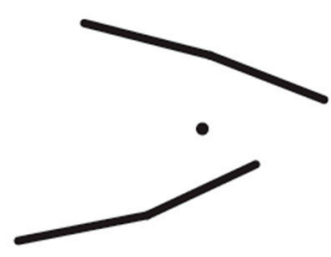

F

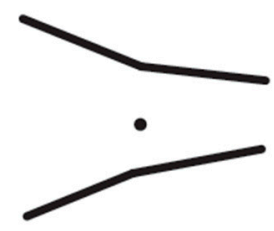

C

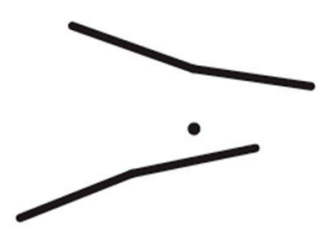

G

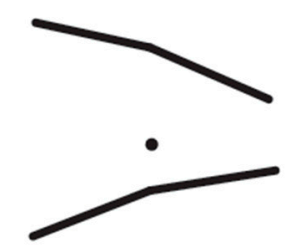

D

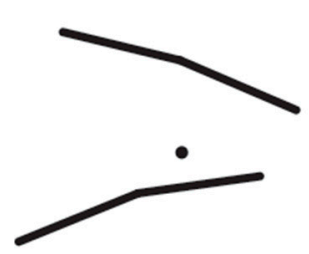

$\mathrm{H}$

Figure 3. The figures used in Experiment 2 (drawn to one-third scale).

The method and observation distance were the same as in Experiment 1. All eight figures were presented rotating around a central vertical axis in parallel projection. There were initially 18 observers from a similar population as those used in Experiment 1 and ignorant concerning the rationale of the experiment. Three observers were rejected because they did not spontaneously report fragmentation in the pretest with the basic two-line Figure 2A. Thus 15 observers completed the experiment. 
Stimuli were presented in a different random order for each observer. All figures were also presented for a second time to each observer in a different random order. The mean of the two presentations for each condition for each observer was used in the analysis.

\subsection{Results}

The means and standard errors of the fragmentation times for each condition are shown in Figure 4. Planned contrasts were carried out on the non-offset Figure 3A-D to test for an effect of complex symmetry. The mean fragmentation time for the more complex (redundant) symmetric Figure 3B,C were found to be significantly lower than for the simple symmetric Figure $3 \mathrm{~A}$ and the asymmetric Figure $3 \mathrm{D}(\mathrm{F}=8.83, \mathrm{df} 1, p<0.005)$. A second planned contrast was carried out to examine the effect of offset. Offset was found to have a significant effect $(\mathrm{F}=8.1, \mathrm{df} 1, p<0.005)$. The interaction between figure and offset was not significant.

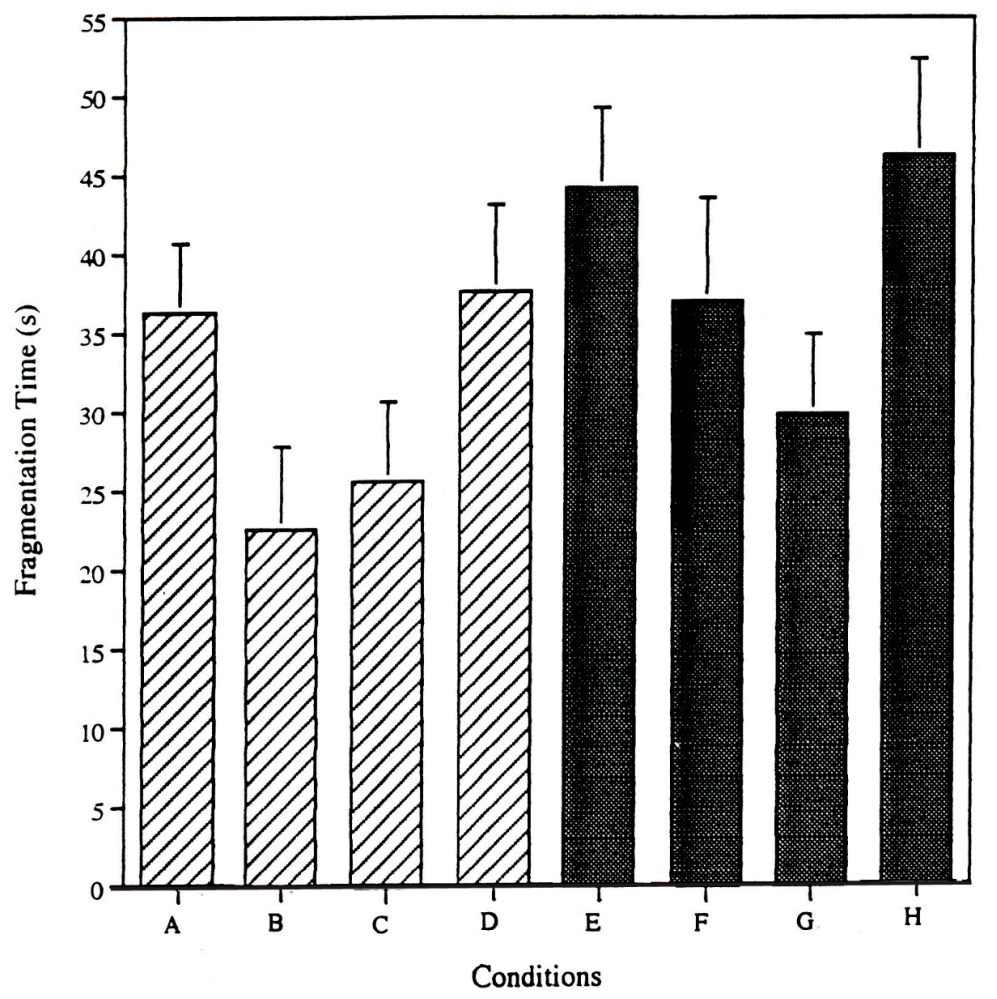

Figure 4. Means and standard errors for each figure used in Experiment 2.

\subsection{Discussion}

The hypothesis was again supported that horizontal symmetry supports grouping more effectively as it becomes more redundant. We also found a very general breakdown of grouping with horizontal offset of the elements. It is an ecological fact that the prevalence of vertically aligned contour edges is ubiquitous in the carpentered world. Our data suggest that it is also a grouping principle.

The data support the view that redundant symmetry is stronger evidence for structure than non-redundant symmetry. The more complex the symmetry, the greater the coincidence it would be for the symmetric layout to arise from a chance arrangement than from intrinsic structure. The visual system could have such contingencies built in to its responses either by evolution or learning. This argument could be made more strongly if in Experiment 1, symmetric (reflected) lines composed of four orientations (Figure 2C) had produced significantly more grouping than those composed of two lines (Figure 2B). Although there was a difference, the fact that it was not significant suggests that the grouping process may have a low ceiling with respect to symmetry redundancy. 
As mentioned in the introduction, the explanation we have proposed could also be couched in Bayesian terms.

\section{Conclusions}

Our past research showed that horizontal symmetry for a simple stimulus was ineffective in producing contour grouping. It appears from the present research that unlike vertical symmetry, horizontal symmetry requires a more complex line to be reflected to produce strong grouping. Barlow and Reeves [3] pointed out that symmetry could produce considerable processing advantages to an organism by allowing subsequent processes to deal with larger units. Gillam [20] pointed out the processing advantages of grouping in the processing of depth and motion. However, until the present studies, there is no direct evidence that symmetry produces grouping outside of figure-ground studies. The present data, however, provide evidence that symmetry results in spatio-temporal grouping if the elements reflected are sufficiently complex. This represents processing consequences of symmetry such as were foreshadowed by Barlow and Reeves [3] and shown for other stimulus factors by Gillam and colleagues (op. cit.). It does not seem likely that the grouping found for complex symmetry was occurring on other grounds.

Acknowledgments: This research was carried out when the author was on the Faculty of The State College of Optometry, SUNY, New York, NY, USA. It was supported by a grant from the U.S. National Institute of Mental Health (R01-MH30840). The author wishes to thank Tarryn Balsdon for help with the figures.

Conflicts of Interest: The author has no conflicts of interest with respect to this paper.

\section{References}

1. Koffka, K. Principles of Gestalt Psychology; Harcourt, Brace \& World: New York, NY, USA, 1935.

2. Treder, M.S.; van der Helm, P.A. Symmetry versus repetition in cyclopean vision: A microgenetic analysis. Vis. Res. 2007, 47, 2956-2967. [CrossRef] [PubMed]

3. Barlow, H.B.; Reeves, B.C. The versatility and absolute efficiency of detecting mirror symmetry in random dot displays. Vis. Res. 1979, 19, 783-793. [CrossRef]

4. Royer, F. Detection of symmetry. J. Exp. Psychol. Hum. Percept. Perform. 1981, 7, 1186-1210. [CrossRef] [PubMed]

5. Wenderoth, P. The salience of vertical symmetry. Perception 1984, 23, 221-236. [CrossRef] [PubMed]

6. Tyler, C.W. Empirical aspects of symmetry perception. Spat. Vis. 1995, 9, 1-7. [CrossRef] [PubMed]

7. Wagemans, J. Characteristics and models of human symmetry detection. Trends Cogn. Sci. 1995, 9, 9-32. [CrossRef]

8. Gurnsey, R.; Herbert, A.M.; Kenemy, J. Bilateral symmetry embedded in noise is detected only at fixation. Vis. Res. 1998, 38, 3795-3803. [CrossRef]

9. Locher, P.J.; Wagemans, J. Effect of element type and spatial grouping on symmetry detection. Perception 1993, 22, 565-587. [CrossRef] [PubMed]

10. Bertamini, M.; Friedenberg, J.D.; Kubovy, M. Detection of symmetry and perceptual organization: The way a lock and key process works. Acta Psychol. 1997, 95, 119-140. [CrossRef]

11. Pomerantz, J.R.; Kubovy, M. Chapter 36 Theoretical Approaches to Perceptual Organization: Simplicity and Likelihood Principles. In Handbook of Perception and Performance; Boff, K.R., Kaufman, L., Thomas, J.P., Eds.; Wiley: New York, NY, USA, 1986; Volume 2.

12. Bahnsen, P. Eine Untersuchung über Symmetrie und Asymmetrie bei visuellen Wahrnehmungen. Z. für Psychol. 1928, 108, 129-154.

13. Rubin, E. Synsoplevede Figurer; Gyldendal: Copenhagen, Denmark, 1915.

14. Peterson, M.A.; Gibson, B.S. Must figure-ground organization precede object recognition? Psychol. Sci. 1994, 5, 253-259. [CrossRef]

15. Mojica, A.J.; Peterson, M.A. Display-wide influences on figure-ground perception: The case of symmetry. Attn. Percept. Psychopys. 2014, 76, 1069-1084. [CrossRef] [PubMed]

16. Kanizsa, G.; Gerbino, W. Convexity and Symmetry in Figure-Ground Organization. In Vision and Artifact; Henle, M., Ed.; Springer: New York, NY, USA, 1976; pp. 25-32. 
17. Machilsen, B.; Pauwels, M.; Wagemans, J. The role of vertical mirror symmetry in visual shape detection. J. Vis. 2009, 9, 1-11. [CrossRef] [PubMed]

18. Gillam, B.; McGrath, D. Orientation relative to the retina determines perceptual organization. Percept. Psychophys. 1979, 26, 177-181. [CrossRef]

19. Feldman, J. Formation of visual "objects" in the early computation of spatial relations. Percept. Psychophys. 2007, 69, 816-827. [CrossRef] [PubMed]

20. Gillam, B. Perceived common rotary motion of ambiguous stimuli as a criterion of perceptual grouping. Percept. Psychophys. 1972, 11, 99-101. [CrossRef]

21. Eby, D.W.; Loomis, J.M.; Solomon, E.M. Perceptual linkage of multiple objects rotating in depth. Perception. 1989, 18, 427-444. [CrossRef] [PubMed]

22. Wallach, H.; O'Connell, D.N. The kinetic depth effect. J. Exp. Psychol. 1953, 65, 205-217. [CrossRef]

23. Gillam, B. Separation relative to length determines the organization of two lines into a unit. J. Exp. Psychol. Hum. Percept. Perform. 1981, 7, 884-889. [CrossRef] [PubMed]

24. Gillam, B.; Grant, T., Jr. Aggregation and unit formation in the perception of moving collinear lines. Perception 1984, 13, 659-664. [CrossRef] [PubMed]

25. Gillam, B.; Broughton, R. Motion capture by a frame: Global or local processing. Percept. Psychophys. 1991, 49,547-550. [CrossRef] [PubMed]

26. Gillam, B. Observations on associative grouping: In honor of Jacob Beck. Spat. Vis. 2005, 18, 147-157. [CrossRef] [PubMed]

27. Rock, I. The Logic of Perception; MIT Press: Cambridge, MA, USA, 1993.

28. Barlow, H. Redundancy reduction revisited. Netw. Comput. Neural Syst. 2001, 12, 241-253. [CrossRef]

29. Csatho, A.; van der Vloed, G.; van der Helm, P.A. Blobs strengthen repetition but weaken symmetry. Vis. Res. 2003, 43, 993-1007. [CrossRef]

30. Baylis, G.C.; Driver, J. Perception of symmetry and repetition within and across visual shapes: Part-descriptions and object based attention. Vis. Cogn. 2001, 8, 163-196. [CrossRef]

31. Makin, A.D.J.; Wright, D.; Rampon, G.; Palumbo, L.; Guest, M.; Sheehan, R.; Cleaver, H.; Bertamini, M. An electrophysiological index of perceptual goodness. Cereb. Cortex 2016, 26, 4416-4434. [CrossRef] [PubMed] 